\title{
HUBBLE SPACE TELESCOPE IMAGING OF GLOBULAR CLUSTERS IN TWO FACE-ON LOW SURFACE BRIGHTNESS GALAXIES UGC 5981 AND UGC 6614
}

\author{
Ji HoOn KIM \\ Center for the Exploration of the Origin of the Universe, Department of Physics and Astronomy, \\ Seoul National University \\ E-mail : jhkim@astro.snu.ac.kr \\ (Received February 28, 2011; Revised August 5, 2011; Accepted August 9, 2011)
}

\begin{abstract}
We present a study searching for globular cluster systems (GCSs) of two face-on low surface brightness galaxies (LSBGs), UGC 5981 and UGC 6614. Based on HST WFPC2 images of F555W and F814W filters, we detect 12 and 18 GC candidates for UGC 5981 and UGC 6614, respectively. Although these two LSBGs have very different bulge properties, they have very similar specific frequencies $\left(S_{N}\right)$ of 0.1 . However, $S_{N} \sim 0.1$ is quite small even for their late morphological types, albeit within errors. This suggests that LSBGs have had star formation histories lacking dominant initial starburst events while accumulating their stellar masses through sporadic star formation activities.
\end{abstract}

Key words : galaxies: individual(UGC 5981, UGC 6614) — galaxies: formation — galaxies: star clusters

\section{INTRODUCTION}

Globular clusters (GCs) in extragalactic systems have long been used to trace star formation history (SFH), dynamics, and structure of their host galaxies (Larson 1992; Ashman \& Zepf 1998). GCs are useful chronometers for timing the chemical evolution of galaxies; their luminosities and colors contain information about their ages and metallicities. So far, most of the observational studies have been centered around globular cluster systems (GCSs) of elliptical galaxies, in which GCSs are easily identified against the smooth, dust-free background. These studies have not only shown the characteristic properties of GCSs, but also featured the characteristic changes of these properties in galaxies. However, our knowledge of GCSs in spiral galaxies still needs to be improved (Harris 1991; Kissler-Patig et al. 1999; Goudfrooij et al. 2003) though, it has been vastly improved due to recent observations of the Hubble Space Telescope.

So far, the Milky Way GCSs has been playing the role of the only standard based on which the GCSs of other galaxies are interpreted. Yet it remains unclear how typical the Milky Way GCSs are either for metal rich early types or for very late types. The properties of GCSs accross all Hubble Types are an improtant probe and test of hierarchical galaxy formation theroy, especially if mergers play an important role in triggering the formation of GCSs and the buildup of spheroidal components.

In the hierarchical picture, one might expect the specific frequency of GCSs to correlate with bulge fraction and/or environment. In a monolithic collapse picture, these properties may be unrelated, while in a delayed formation scenario, one might expect a rather low specific frequency.

GCSs have been detected in all nearby massive high surface brightness (HSB) galaxies and in many dwarf galaxies (Harris 1991; Miller et al. 1998) and seem to form naturally in many types of galaxies. However, the formation epoch and mechanism of GCSs are still unknown. Candidate scenarios are: 1) a pre-galactic formation (Peebles \& Dicke 1968), 2) the formation during the turbulent early collapse of the galaxy from primordial fragments or in supergiant molecular clouds (Searle \& Zinn 1978; Harris \& Pudritz 1994), and 3) the formation due to perturbations induced by mergers or accretion (Ashman \& Zepf 1992). While many cases of young GCSs in merging and interacting galaxies are now known (e.g., Schweizer 1997), it is still believed that most of the GCs were formed at early time (Chaboyer et al. 1996).

Low surface brightness (LSB) galaxies make interesting test cases. One view is that LSB galaxies have been forming continuously over a Hubble time, but, as they are the most isolated type of spiral galaxies, have had suppressed star formation (Bothun et al. 1993; Mo et al. 1994). An alternative view to explain low surface brightness, low abundance, and blue colors in these galaxies is that they simply have delayed star formation histories (SFHs) (Impey \& Bothun 1989; McGaugh, \& Bothun 1994). Recently, Kim (2007) and Kim et al. (in preparation) suggest that LSBGs may have been experiencing sporadic star formation activities along Hubble time due to late gas infall based on their stellar 

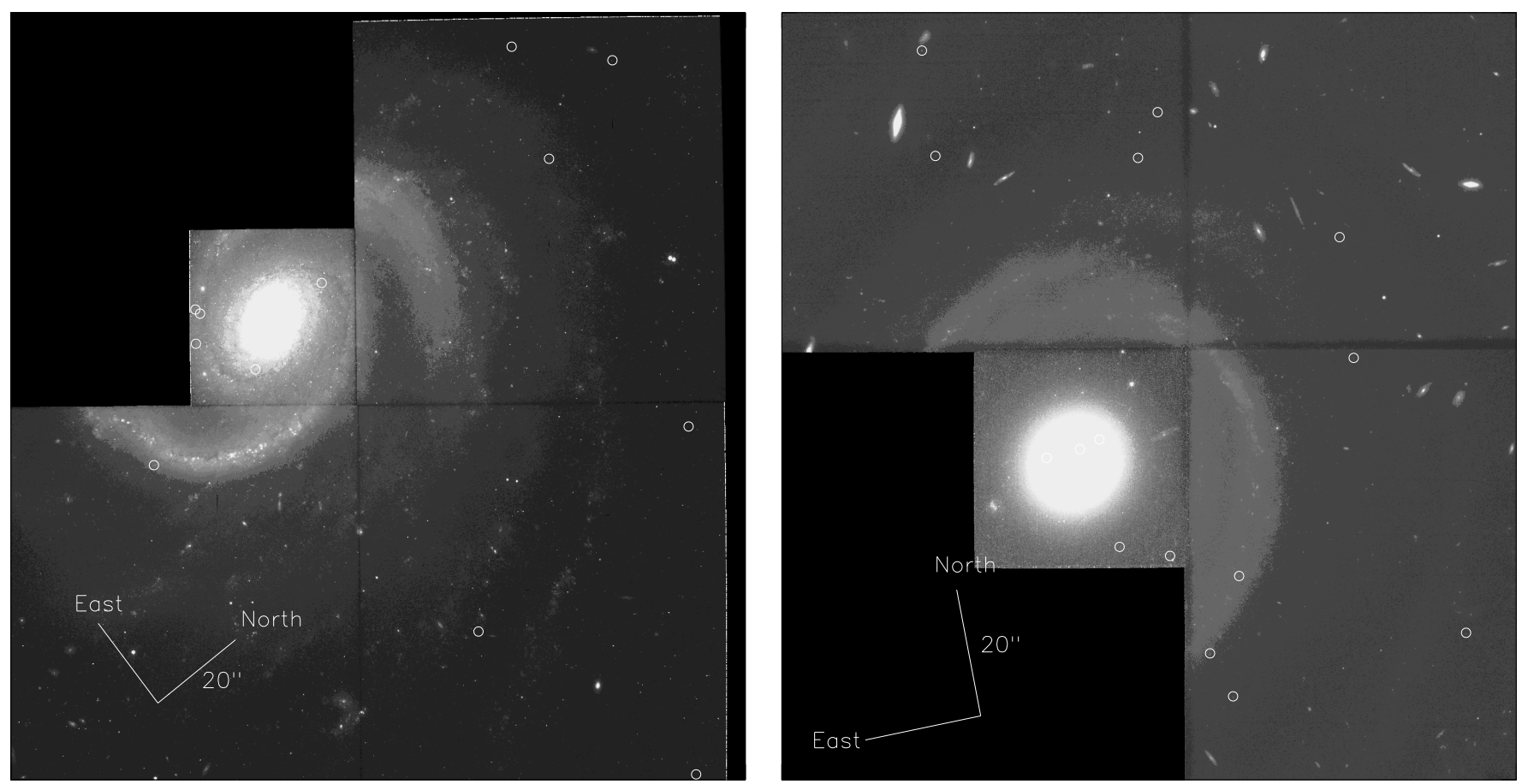

Fig. 1.- A mosaic image of UGC 5981. No dithering is performed for this galaxy. The image is overlapped with the locations of GC candidates which are marked by circles. The orientation of the image is indicated by the L-shape mark each side of which has the length of 20 arcseconds.

masses and star formation rates. These scenarios can be tested by the nature of GCSs within LSB galaxies.

Despite the fact that GCSs can be good chronometers to put constraints on the formation epochs of LSBGs, there has not been much work done on the GCSs of LSB galaxies. This is mainly due to the fact that many of the most massive and interesting LSB galaxies have too large distances to detect their GCSs from the ground. Yet, there have been a couple of studies of GCSs within LSBGs recently. Sharina et al. (2005) searched for GCSs within 57 LSB dwarf/irregular galaxies and found GC candidates from $50 \%$ of their sample. Puzia \& Sharina (2008), using spectroscopy on GC candidates of five LSB dwarf galaxies among the sample of Sharina et al. (2005) confirm that 26 out of $27 \mathrm{GC}$ candidates are genuine and show that the specific frequencies of these galaxies are well matched with dwarf galaxies found in nearby local clusters while metallicities of these GCSs are a bit low, except for an isolated S0 galaxy. Villegas et al. (2008) tried to find GCSs of six LSB spiral galaxies which turn out to have a rather wide range of central surface brightnesses. Two LSB spiral galaxies among their sample still have numerous GC candidates and have specific frequency values around 2.0.

However, these LSB galaxies studied by the aforementioned studies are not "typical" LSB galaxies which
Fig. 2.- A drizzled image of UGC 6614. For the drizzling procedure, e followed the general guide line given by Koekemoer et al. (2002). The image is also overlapped by the locations of GC candidates which are marked by circles. The orientation is also given by the L-shape mark as with Fig. 1.

have distinctively different characteristics from normal spiral galaxies. In order to diagnose the unique nature of LSBGs, it is critical to probe GCSs of LSB spiral galaxies. The high spatial resolution and deep imaging capability of HST make it possible to detect GCSs in these massive "Malin"-type LSBs (Bothun, Impey, Malin, \& Mould 1987), and it could be significant to just confirm whether LSBs have systems of old GCs.

In this paper, we present an imaging study of the GCSs of two face-on LSB spiral galaxies, UGC 5981 and UGC 6614. In Section 2, we present the properties of the target galaxies and the observations, and describe the photometry involved. In Section 3 the results are analyzed. In Section 4 we compare the GCSs to each other. Summary is presented in Section 5 .

\section{OBSERVATION AND DATA REDUCTION}

\subsection{Observations}

\subsubsection{The Target Galaxies}

We obtained available WFPC2 observations of two face-on LSB spiral galaxies, UGC 5981 and UGC 6614 (Program GO 8213). Basic properties of the two galaxies are given in Table 1, and composites of our images are shown in Fig. 1 and Fig. 2. 
Table 1.

Basic Properties of UGC 5981 and UGC 6614

\begin{tabular}{cccc}
\hline \hline Properties & UGC $5981^{\mathrm{a}}$ & UGC 6614 & Reference \\
\hline R.A. $(2000)$ & $10^{h} 52^{m} 03.5$ & $11^{\mathrm{s}} 39^{m} 11^{\mathrm{s} .7}$ & 1,2 \\
Dec. $(2000)$ & $+10^{\circ} 09^{\prime} 00^{\prime \prime}$ & $+17^{\circ} 08^{\prime} 22^{\prime \prime}$ & 1,2 \\
Type & $\mathrm{Sc}$ & $\mathrm{Sa}$ & 2 \\
$B_{T}^{0}$ & $12.17 \pm 0.19$ & $14.11 \pm 0.3$ & 1 \\
$(B-V)_{T}^{0}$ & & $0.80 \pm 0.03$ & 1 \\
Inclination & $37^{\circ}$ & $34^{\circ}$ & 3,4 \\
Position angle & $46^{\circ}$ & $330^{\circ}$ & $3,^{\mathrm{b}}$ \\
$v_{\text {helio }}$ & $2719 \pm 4 \mathrm{~km} \mathrm{~s} \mathrm{~m} \mathrm{~s}^{-1}$ & $8351 \pm 20 \mathrm{~km} \mathrm{spc}$ & 1 \\
Adopted distance & $36.3 \mathrm{Mpc}$ & 34.6 & $\mathrm{c}$ \\
$(m-M)$ & 32.8 & -20.5 & $\mathrm{~d}$ \\
$M_{B_{T}^{0}}$ & -20.6 & & $\mathrm{e}$ \\
\hline
\end{tabular}

\footnotetext{
${ }^{a}$ Also NGC 3433

${ }^{\mathrm{b}}$ Estimated from our image.

${ }^{\mathrm{c}}$ Derived from the above $v_{\text {helio }}$ values using $\mathrm{H}_{0}=75 \mathrm{Km} \mathrm{s}^{-1} \mathrm{Mpc}^{-1}$

${ }^{\mathrm{d}}$ Derived from the above distance.

${ }^{\mathrm{e}}$ Derived from the above values.
}

References.-(1) de Vaucouleurs et al. (1991) (2) Nilson (1973) (3) Canzian (1998) (4) McGaugh (1994).

UGC 5981 is classified as an Sc galaxy (de Vaucouleurs et al. 1991, hereafter RC3; Nilson 1973; Materne 1978) with an inclination angle of $37^{\circ}$ (Canzian 1998). It is at a distance of $36 \mathrm{Mpc}$ based on its heliocentric velocity of $2719 \mathrm{~km} \mathrm{~s}^{-1}$ and the assumed Hubble constant of $75 \mathrm{~km} \mathrm{~s}^{-1}$. UGC 6614 is classified as an Sa galaxy (de Vaucouleurs et al. 1991; Nilson (1973)), with an inclination angle of $34^{\circ}$ (McGaugh, \& Bothun 1994), it is at a distance of $85 \mathrm{Mpc}$, as calculated from its heliocentric velocity of $6351 \mathrm{~km} \mathrm{~s}^{-1}$ and the Hubble constant.

The two galaxies have very similar luminosities, but are chosen from the samples of Knezek (1993) and McGaugh, \& Bothun (1994) to have different bulge properties. UGC 6614 is the nearest "Malin"-type massive LSB galaxy with a prominent bulge and a very extended LSB disk. Even with a central disk surface brighness of $B=24.28 \mathrm{mag} \operatorname{arcsec}^{-2}, M_{B}=-20.5$ for $\mathrm{H}_{0}=75 \mathrm{~km} \mathrm{~s}^{-1}$. The R-band scale length of $9.2 \mathrm{kpc}$ is also about thrice as large as a typical HSB spiral galaxy. On the other hand, UGC 5981 has a moderate bulge.

\subsubsection{The Observations}

We observed UGC 5981 and UGC 6614 with the WFPC2 on the Hubble Space Telescope (HST). As shown in Fig. 1 and Fig. 2, we aligned the pointings so that the bulges of the target galaxies are placed rea- sonably well within the PC chip. Images are obtained in the F555W and F814 filters for both galaxies.

For UGC 5981, images were obtained with total integration times ranging from 1200 to 1300 s. For each filter, two exposures were taken without a shift.

For UGC 6614, four dithered positions were taken for each filter with shifts ranging from 0 ". 45 to 0. "60. For each dither position, two exposures were obtained with total integration times ranging from 1100 s to 1300 s.

\subsection{Data Reduction}

\subsubsection{Basic Reduction and Photometry}

The basic image reduction was carried out under IRAF. Since only UGC 6614 images were dithered, drizzling process is only applied for UGC 6614. For UGC 5981, two science images for each band were simply combined with the task CRREJ, which rejected the vast majority of cosmic rays. For UGC 6614, we followed the general guideline given in "The Dither Handbook" (Koekemoer et al. 2002). Using tasks in the DITHER package, sky backgrounds were subtracted and offsets were determined. After creating static bad pixel masks and cosmic ray masks, final drizzled images were constructed using the DRIZZLE task in the DITHER package.

The photometry was carried out using the SEX- 
Table 2.

GC candidates in UGC 5981

\begin{tabular}{ccccc}
\hline \hline ID & R.A. $(2000)$ & Dec. $(2000)$ & V $(\mathrm{mag})$ & $(V-I)(\mathrm{mag})$ \\
\hline KM 5981-1 & $10: 52: 03.52$ & $+10: 08: 44.88$ & 26.92 & 1.49 \\
KM 5981-2 & $10: 52: 03.86$ & $+10: 09: 05.86$ & 27.10 & 1.61 \\
KM 5981-3 & $10: 52: 04.65$ & $+10: 08: 42.44$ & 26.10 & 0.74 \\
KM 5981-4 & $10: 52: 04.29$ & $+10: 08: 38.43$ & 26.88 & 1.33 \\
KM 5981-5 & $10: 52: 04.57$ & $+10: 08: 42.70$ & 26.69 & 1.36 \\
KM 5981-6 & $10: 52: 03.38$ & $+10: 08: 17.15$ & 26.67 & 1.48 \\
KM 5981-7 & $10: 51: 58.91$ & $+10: 08: 49.20$ & 27.14 & 1.71 \\
KM 5981-8 & $10: 51: 55.58$ & $+10: 09: 06.92$ & 27.27 & 1.45 \\
KM 5981-9 & $10: 51: 59.26$ & $+10: 09: 47.42$ & 27.09 & 1.75 \\
KM 5981-10 & $10: 52: 03.71$ & $+10: 10: 19.12$ & 26.84 & 1.55 \\
KM 5981-11 & $10: 52: 03.23$ & $+10: 09: 57.08$ & 26.57 & \\
KM 5981-12 & $10: 52: 04.71$ & $+10: 10: 04.60$ & 27.13 &
\end{tabular}

Note.- Units of right ascension are hours, minutes, and seconds, and units of declination are degrees, arcminutes, and arcseconds.

Table 3.

GC candidates in UGC 6614

\begin{tabular}{ccccc}
\hline \hline ID & R.A. (2000) & Dec. $(2000)$ & V $(\mathrm{mag})$ & $(V-I)(\mathrm{mag})$ \\
\hline KM 6614-1 & $11: 39: 14.53$ & $+17: 08: 39.49$ & 29.62 & 1.90 \\
KM 6614-2 & $11: 39: 14.77$ & $+17: 08: 38.61$ & 29.27 & 1.93 \\
KM 6614-3 & $11: 39: 15.16$ & $+17: 08: 38.35$ & 29.01 & 1.70 \\
KM 6614-4 & $11: 39: 14.54$ & $+17: 08: 22.46$ & 28.72 & 1.99 \\
KM 6614-5 & $11: 39: 13.99$ & $+17: 08: 19.46$ & 28.62 & 1.84 \\
KM 6614-6 & $11: 39: 13.76$ & $+17: 08: 03.32$ & 27.41 & 1.32 \\
KM 6614-7 & $11: 39: 13.59$ & $+17: 07: 56.02$ & 27.78 & 1.33 \\
KM 6614-8 & $11: 39: 13.27$ & $+17: 08: 14.18$ & 27.69 & 1.75 \\
KM 6614-9 & $11: 39: 11.53$ & $+17: 08: 43.79$ & 28.34 & 1.92 \\
KM 6614-10 & $11: 39: 08.32$ & $+17: 08: 26.06$ & 28.04 & 0.84 \\
KM 6614-11 & $11: 39: 10.86$ & $+17: 07: 58.26$ & 28.34 & 1.93 \\
KM 6614-12 & $11: 39: 11.43$ & $+17: 09: 02.66$ & 28.64 & 1.57 \\
KM 6614-13 & $11: 39: 13.19$ & $+17: 09: 27.55$ & 28.53 & 1.32 \\
KM 6614-14 & $11: 39: 13.51$ & $+17: 09: 21.20$ & 27.73 & 1.58 \\
KM 6614-15 & $11: 39: 17.78$ & $+17: 09: 21.72$ & 27.55 & 1.40 \\
KM 6614-16 & $11: 39: 15.26$ & $+17: 10: 03.28$ & 27.82 & 1.87 \\
KM 6614-17 & $11: 39: 15.76$ & $+17: 09: 27.98$ & 28.30 & 27.40 \\
KM 6614-18 & $11: 39: 15.68$ & $+17: 09: 44.48$ & & \\
\hline
\end{tabular}

Note.-Units of right ascension are hours, minutes, and seconds, and units of declination are degrees, arcminutes, and arcseconds. 
TRACTOR software (Bertin \& Arnouts 1996). The finding parameters were set to two connected pixels 2.5 $\sigma$ above the local background (computed in a $10 \times 10$ pixel mesh). In order to avoid being affected by intense background fluctuations, the photometry was intended to be carried out with small radius apertures. However, too small radii can cause unstable photometry. While measuring aperture correction, we have performed extensive photometry experiments with various radii. We decided to carry out 2 pixel radius aperture photometry for UGC 5981 based on these results. The FWHMs in pixels of the PSFs for the PC and the WF chips are very similar in spite of a difference pixel scale of over a factor of 2 . Therefore, the same aperture system was used for all chips. The same aperture system was used for a number of similar studies (Miller et al. 1997; Kissler-Patig 1999; Goudfrooij et al. 2003).

We then performed 4 pixel radius photometry for UGC 6614 instead of 2 pixel radius photometry as the linear scale for this galaxy is enlarged by a factor of 2 through drizzling process. Surely the FWHMs on chips should be smaller than 4 pixels compared to those of UGC 5981 smaller than 2 pixels. But, as too small an aperture can lead to errors due to centering problems with the undersampled PSF and spatial variations of the PSF, 4 pixel radius aperture was used.

Corrections to get the equivalent magnitude to a 0.5 aperture were determined by measurements with various radii of isolated bright objects on the images and found to be 0.837 and $0.300 \mathrm{mag}$ for the PC and WF in the F555W filter, respectively, and 0.701 and 0.304 mag in the F814W filter, for UGC 5981. For UGC 6614, these corrections are 0.450 and $0.296 \mathrm{mag}$ for the PC and WF, respectively in the F555W filter, and 0.802 and $0.352 \mathrm{mag}$ in the $\mathrm{F} 814 \mathrm{~W}$ filter.

Lastly, the measured WFPC STMAG magnitudes F555W and F814W were converted into JohnsonCousins $\mathrm{V}$ and I magnitudes using relationships as given by Holtzman et al. (1995) and Goudfrooij et al. (2001). The conversion relationships are:

$$
\begin{gathered}
V=F 555 W+0.643+0.004 \times(F 555 W-F 814 W)- \\
0.015 \times(F 555 W-F 814 W)^{2} \\
I=F 814 W+(-1.266)+0.018 \times(F 555 W- \\
F 814 W)+0.016 \times(F 555 W-F 814 W)^{2}
\end{gathered}
$$

\subsubsection{Globular Cluster Selection}

Given that the SEXTRACTOR software detected about 2000 objects per chip originally, an automated selection process was necessary. Besides photometric data, structural parameters such as FWHM and stellarity index were good candidates to use among the parameters given by SEXTRACTOR.

Along with the FWHM in pixels, Miller et al. (1997) used the difference in F555W magnitude between an aperture of 0.5 pixel radius and an aperture of 3.0 pixel radius (hereafter denoted by $\Delta_{V}(0.5-3)$ ) for NGC 7252 , which lies at a distance of $64.4 \mathrm{Mpc}$. This selec- tion criterion was developed based on clusters detected in WFPC2 observations of the elliptical galaxy NGC 1700 Whitmore et al. (1997). Miller et al. (1997) found a similar distribution of types in an independent check and tests on other galaxies in their sample gave very similar results.

With slightly more or less distances B of our galaxies, especially UGC 5981 with very similar data quality, $\Delta_{V}(0.5-3)$ makes a good selection criterion candidate. According to Miller et al. (1997), objects with $\Delta_{V}(0.5-3)>2.4$ or $F W H M>2.5$ pixels are classified as diffuse. Objects with $F W H M<1.0$ pixel also are ignored as they are most likely hot pixels or residual cosmic rays. Objects with $\Delta_{V}(0.5-3)<1.0$ in the $\mathrm{PC}$ and objects with $\Delta_{V}(0.5-3)<0.5$ in the WF are also excluded.

These criteria on FWHM agree quite well with those from Kissler-Patig et al. (1999). They carried out extensive experiments with artificial point sources and artificial GCs. For the latter, they used WFPC2 point spread functions obtained with TINY TIM (Krist \& Hook 1997) and, convolved with a Modified Hubble law of 0.1 and $0^{\prime \prime} .2$. The resulting FWHMs of their artificial objects range from 0.8 to 1.5 pixels, with 1.5 to 2.5 pixels for artificial stars and GCs, respectively, at $\mathrm{B}<23.5$. They defined robust selection criteria for globular clusters based on the FWHM of the detected objects $(>0.7$ and $<3.0$ pixels, as returned by SEXTRACTOR).

Since at the distance of our galaxies, GC are only marginally resolved or unresolved, we need to introduce further selection criteria to distinguish stars from cluster candidates. Most observations of GCSs in the Milky Way and nearby galaxies show that even the most metal-rich GCSs have $(V-I)<1.5$ (Peterson 1993; Whitmore \& Schweizer 1995). On the other hand, many field stars in the Galaxy are likely to be much redder than this. Hence, we introduce a fairly generous selection criterion for the color: $(V-I)<2$, to prevent the exclusion of reddened clusters. For the lower limit, we set $0<(V-I)$, but generally cluster candidates have the color of $0.5<(V-I)$.

On top of the color cut, we also introduced the upper limit of magnitude. Some GCs can be as bright as $M_{V}=-12$, if not easily. We found that none of our cluster candidates reach to $M_{V}=-10$.

\section{NUMBERS OF GLOBULAR CLUSTERS AND SPECIFIC FREQUENCIES}

\subsection{Globular Cluster Candidates}

Final samples of 12 and 18 GC candidates in UGC 5981 and UGC 6614, respectively (see Tables 2 and 3) were selected. The distribution of globular candidates is shown in Fig. 1 and Fig. 2, respectively. Note that among the cluster candidates, there are a number of candidates which are projected on spiral arms. While it is obvious that some of them are associated with 
spiral arms for sure, not every candidate projected on spiral arms should be actually in spiral arms. Especially for UGC 5981, quite a few cluster candidates are projected on structures developed from spiral arms, but do not really show any distinctive characteristics from other candidates. While our sample galaxies are fairly face-on, dissociation of real GCs from stellar associates or stellar clusters on spiral arms is not an easy task. Therefore, we include all the cluster candidates projected on spiral structures in the final samples.

Color-magnitude diagrams for the GC candidates in UGC 5981 and UGC 6614 are shown in Fig. 4 and 5, respectively, together with related histograms. The mean colors and dispersion for the samples are $(V-I)=1.45 \pm 0.25$ and $(V-I)=1.65 \pm 0.30$ for UGC 5981 and UGC 6614, respectively. For comparison, the Milky Way GCSs have a peak at $(V-I)=0.95$ corresponding to a metal-poor $([\mathrm{Fe} / \mathrm{H}] \approx-1.5)$ halo population, and another at $(V-I) \approx 1.1$ corresponding to more metal-rich $([\mathrm{Fe} / \mathrm{H}] \approx-0.5)$ thick-disk populations.

\subsection{The Specific Frequencies and the Total Number of Globular Clusters}

The specific GC frequency, $S_{N}$, is the number of clusters per unit galaxy V-band luminosity (normalized to $\left.\left.M_{V}=-15\right) ; S_{N}=N_{G C} 10^{0.4\left(M_{V}+15\right.}\right)$, where $M_{V}$ is the absolute visual magnitude of the parent galaxy Harris et al. 1981). $S_{N}$ was introduced primarily for use in elliptical galaxies, where there is little variation in the stellar population from one galaxy to another. And this is not true for spiral galaxies. $S_{N}$ is correlated with Hubble type such that it increases by a factor of 10 from late type spirals to early type spirals and ellipticals (Harris 1991). Recent studies, including the compilation of Ashman \& Zepf (1998) along with Kissler-Patig et al. (1999) and Goudfrooij et al. (2003), show $\left\langle S_{N}>=0.4 \pm 0.2\right.$ for Sc spirals to 1.3 \pm 0.5 for Sa spirals.

With the $S_{N}=0.4$ for Sc galaxies and $M_{B}=-19.9$, the estimated number of GCs is about 35 for UGC 5981. And for UGC 6614, which is classified as Sa, the estimated number of clusters is about 430 with $S_{N}=$ 1.3 and $M_{V}=-21.3$.

However, our observations do not cover entire galaxies. Therefore, when we calculate $S_{N}$, we need to consider only the covered areas. In order to do this, we measure total visual magnitude of covered areas of UGC 5981 and UGC 6614 by masking foreground and background objects, accumulating all pixel counts inside images, and subtracting skybackground.

As a result, we obtained $V=12.67 \pm 0.05$ and $V=13.62 \pm 0.05$ for covered parts of UGC 5981 and UGC 6614, respectively. From obtained total visual magnitude, we estimate $S_{N}=0.11 \pm 0.005$ and $S_{N}=0.07 \pm 0.005$ for UGC 5981 and UGC 6614, respectively. Note that we did not exclude GC candidates projected on spiral arms.

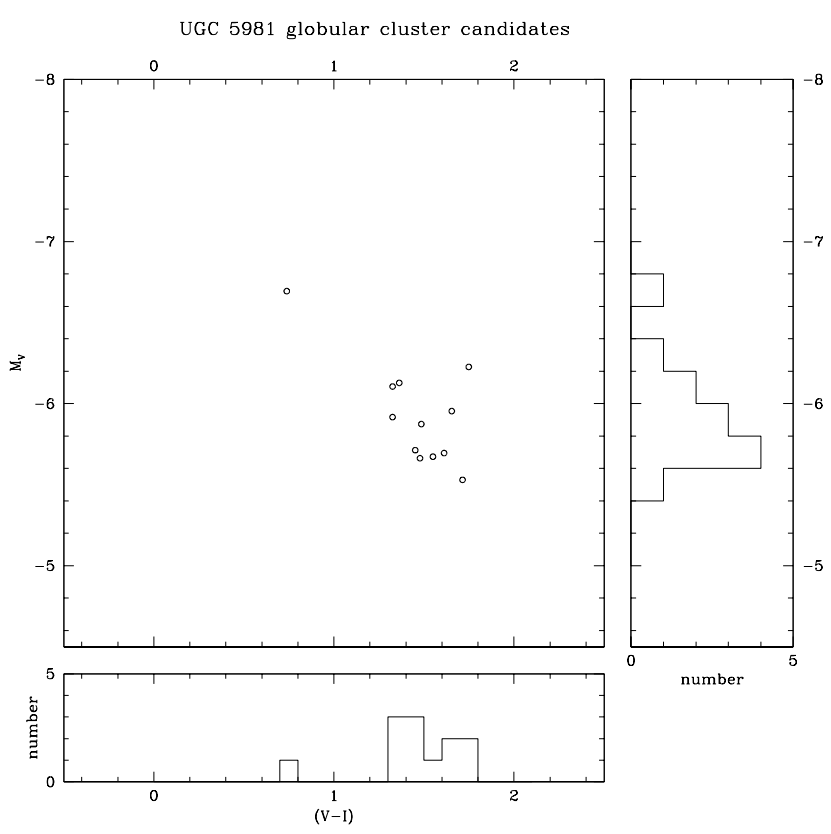

Fig. 3.- Color-magnitude diagram and related histograms for GC candidates of UGC 5981. The abscissa is the $V I$ color, while the ordinate is the absolute magnitude in the $\mathrm{V}$-band $\left(M_{V}\right.$. The histograms of the $V I$ color and $M_{V}$ are presented along with the respective axes.

Then again, one must take into account the opposite side of the galaxies while considering covered areas of the galaxies. LSBGs are believed to have very thin disks. Holwerda et al. (2005) studied the opacity of spiral galaxy disks using HubbleSpaceTelescope archival images and concluded that LSBGs have transparent disks along with Sd type galaxies. In fact, UGC 6614 is one of two LSBGs which are studied by Holwerda et al. (2005). Therefore, we only need to concern the number of GCs blocked by the bulges and any GCs which reside on the the opposite side of disks, which are reddened significantly.

In the worst case, the number of GCs can be as twice as many. However, the reasonable prediction would be that only the number of bulge GC candidates should be extrapolated. As seen in Fig. 1 and Fig. 2, the number of GC candidates located within the bulges of UGC 5981 and UGC 6614 are two and three, respectively. Therefore, if we assume that we miss out as many GC candidates as these, we would have 14 and 21 GC candidates for UGC 5981 and UGC 6614, respectively. Then, $S_{N}$ would not be changed much and would be 0.12 and 0.09 for UGC 5981 and UGC 6614 , respectively. Since we need to assume that some of these candidates are not genuine GCSs, our specific frequency will be our upper limit. 


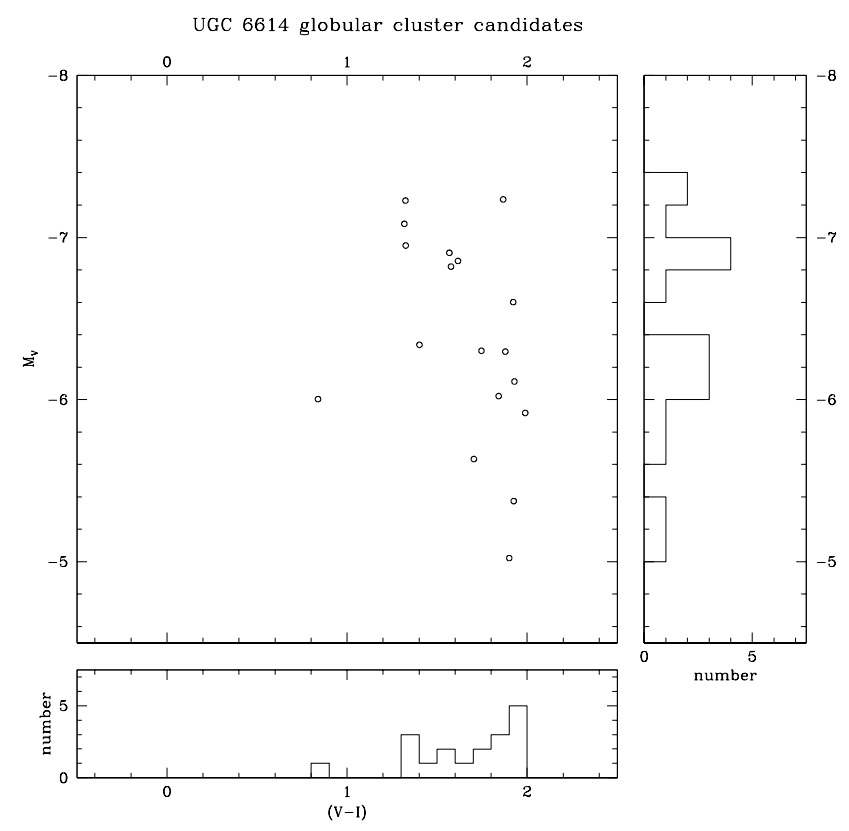

Fig. 4. - Color-magnitude diagram and related histogram for GC candidates of UGC 6614. As with Fig. 3, the abscissa is the $V I$ color and the ordinate is $M_{V}$. The histograms of the $V I$ color and $M_{V}$ are presented along with the respective axes as well.

\section{POPULATIONS AND LUMINOSITY FUNC- TION OF GLOBULAR CLUSTERS}

\subsection{Cluster Populations}

Although there are so few GCs for our sample galaxies that it seems very difficult to speak of their distribution, it does show some resemblance to GCSs of other galaxies. GCs in UGC 5981 have a "flattened" bimodality for $(V-I)$ colors, although the actual distribution is thinned out through the range of color and shifted towards the redder end. Therefore it has a primary peak of $(V-I) \approx 1.4$, instead of $(V-I) \approx 1.1$. Its secondary peak appears at $(V-I) \approx 1.7$, making it very easy to think that $\mathrm{GC}$ candidates are reddened. Yet, considering the lower luminosity limit of candidates acquired, it is not so obvious that GCSs candidates of UGC 5981 are not red and metal-poor.

GCs in UGC 6614 have a more intriguing distribution. The distribution shows a strong peak at $(V-I) \approx 1.9$ and its secondary peak appears around $(V-I) \approx 1.4$. We perversely looked for rejected cluster candidates whose $(V-I)>2$ to check whether there is an evidence of a huge shift towards red color, but could not find any. Therefore, it is hard to explain the odd shape of distribution for the GC candidates of UGC 6614.

\subsection{Luminosity Function of Globular Clusters}

Interestingly, it is UGC 6614 which has a peak at $V \approx-7.3$, at which nearby elliptical galaxies and spiral galaxies have peaks of their Gaussian luminosity function. However, the luminosity function of UGC 6614 is not a Gaussian. Instead, it has a fading tail into less luminous end. And generally, those lower end cluster candidates are redder objects as well. Therefore, the shape of the luminosity function of UGC 6614 is unclear.

On the other hand, the luminosity function of GCs in UGC 5981 has a peak pushed down to $V \approx-6.3$. Although, fitting routine is remained to be done, the luminosity function has a shape of a power-law function. However, power-law luminosity functions are generally associated with young stellar clusters, which have masses and sizes of GCs. And these younger populations generally reside in the LMC and in many starbursting and merging galaxies (e.g., Elson \& Fall 1985; Meurer et al. 1995; Whitmore et al. 1997). And clearly, UGC 5981 does not have many young stellar cluster candidates, which are generally believed to have $(V-I)<0.5$ color. Therefore, close investigation to look for possible missing young cluster candidates is to be done.

\section{DISCUSSION}

\subsection{Comparison between the Two Galaxies}

UGC 5981 and UGC 6614 have similar total luminosities. But, there are significant differences between in the disk and bulge properties of the two galaxies. Therefore, if the formation and evolution of GCs in a spiral galaxy, especially, a low surface brightness spiral galaxy, are subject to the influence of the properties of the disk and bulge of the galaxy, we can expect to find differences in the globular cluster systems of these two galaxies.

UGC 5981 could have been expected to have only a halo population with no or weak presence of metal-rich globular clusters, due to the absence of a prominent bulge (and low metalicity). Yet, it lacks a halo population but not disk clusters, if the characteristics of the Milky Way globular cluster systems holds for low surface brightness spiral galaxies.

UGC 6614 has a prominent bulge and a very extended disk. With a true characteristics of a low surface brightness giant, it has a bit flattened color distribution for bluer GCs with very strong peak of redder clusters. However, given the uncertainties of reddening, we can not classify those globular cluster systems into certain populations. However, considering the relatively metalrich character of giant low surface brightness galaxies, the GCSs of UGC 6614 seem to sustain the general feature of the globular systems of UGC 5981. Therefore, the fact that the GCSs of UGC 5981 and UGC 6614 are very similar despite differences in the disk and bulge 
characteristics, which may suggest that the the process that forms the total GCSs around spiral galaxies are not strongly related to the process that forms the disk and bulge.

\subsection{Comparison with HSB Spiral Galaxies}

Our sample galaxies are low surface brightness galaxies, which means that any significant difference between the GC systems of the two galaxies and other HSB spiral galaxies reveal any differences between the formation and evolution of low surface brightness galaxies and those of higher surface brightness counterparts.

There has been a tremendous expansion of samples in studies of GCSs in spiral galaxies, although we have not reached to the point of considering a census. About 40 galaxies whose morphological types range from Sa to Sdm were searched for GCs by Kissler-Patig et al. (1999), Goudfrooij et al. (2003), Rhode \& Zepf (2003), Chandar et al. (2004), Barmby et al. (2006), Rhode et al. (2007), Proctor et al. (2008), Villegas et al. (2008), Mora et al. (2009), Harris et al. (2009), and Rhode et al. (2010). Listing a few in order to give a good comparison with consistency, $S_{N}$ values are $0.9 \pm 0.2(\mathrm{M} 31$, $\mathrm{Sb}$ ), $0.2 \pm 0.1$ (NGC 253, Sc), $1.7 \pm 0.5$ (NGC 2683, $\mathrm{Sb}$ ), $1.9 \pm 0.2$ (NGC 3628, Sb), 1.1 \pm 0.3 (NGC 4013, $\mathrm{Sb}), 1.2 \pm 0.6(\mathrm{NGC} 4216, \mathrm{Sb}), 0.6 \pm 0.2$ (NGC 4517, $\mathrm{Sc}), 0.6 \pm 0.2(\mathrm{NGC} 4565, \mathrm{Sb}), 1.7 \pm 0.6$ (NGC 4594, $\mathrm{Sa}), 0.9 \pm 0.3$ (NGC 5170, Sb), $0.5 \pm 0.1$ (IC 5176, Sbc),

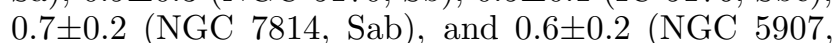
$\mathrm{Sc})$. Therefore, within error, $S_{N}$ values of our two low surface brightness galaxies are not out of range from those of high surface brightness spiral galaxies, especially UGC 5981. However, they still have significantly lower number of GCSs.

We compile $S_{N}$ values of 38 late-type galaxies obtained with these studies and plot them with our values of LSBGs. In Fig. 5, we plot $S_{N}$ against morphological types with the upper panel and against $M_{V}$ with the bottom panel. As expected, both our LSBGs lie lower than their same morphological counterparts. Particularly, UGC 6614, considering its morphological type of $\mathrm{Sa}$, lies much lower than its morphological brethren. Including three Sab type galaxies to make statistics plausible, four Sa type galaxies have the mean $S_{N}$ of 1.18 which is more than order of magnitudes bigger than $S_{N}$ of UGC 6614 . On the other hand, Sc type galaxies, including $\mathrm{Sbc}$ and Scd again, have the mean $S_{N}$ of 0.60 which is much larger than that of UGC 5981 again. However, considering Sc type galaxies only, the mean drops to 0.35 . Even if we accept the $S_{N}$ values acquired by assuming that we miss out GCs due to lack of visibility, LSBGs still have lower $S_{N}$ than HSB galaxies.

On the other hand, UGC 5981 and UGC 6614 have lower $S_{N}$ than late-type galaxies with comparable luminosity as well. The mean $S_{N}$ of the late type galaxies whose $M_{V}$ range between -20 and -21 among those 38
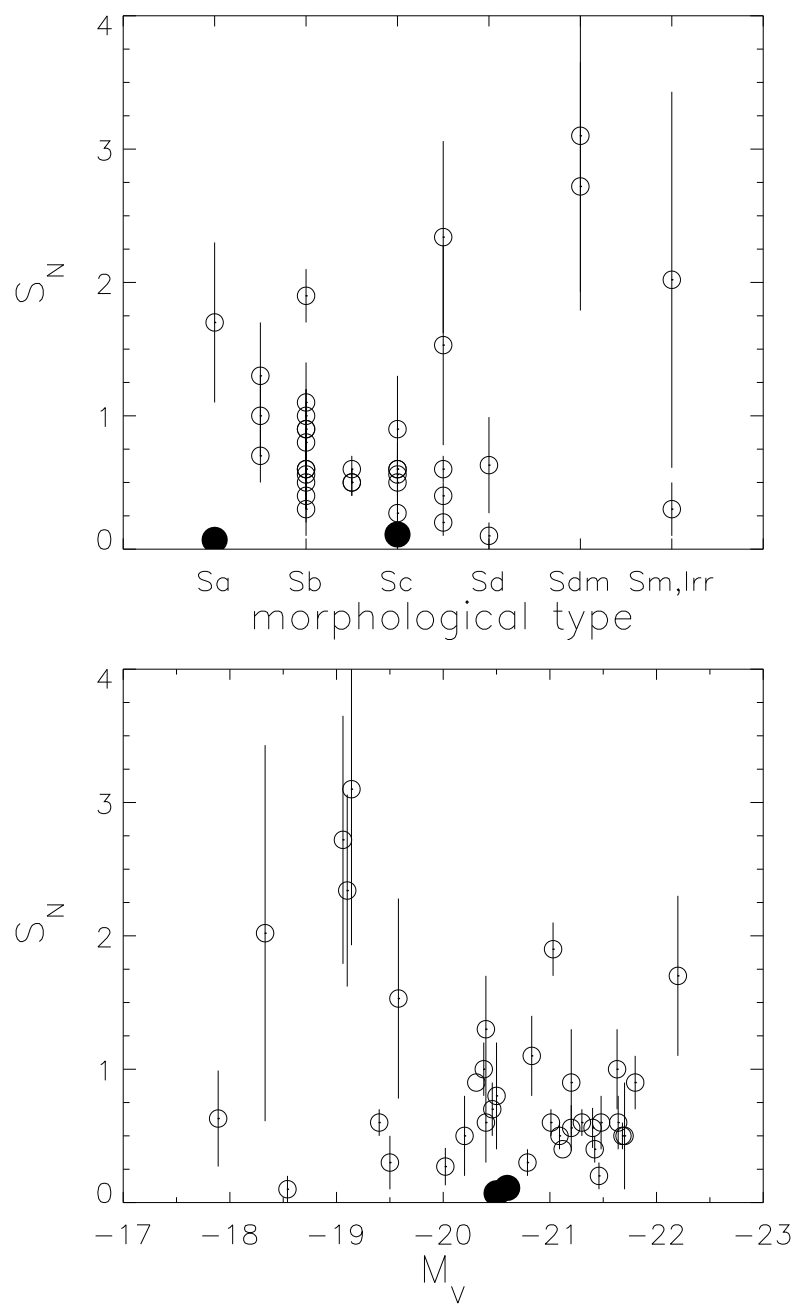

Fig. 5.- Correlations of $S_{N}$ with morphological types and $M_{V}$. The upper panel shows the correlation with morphological types and the bottom panel shows the correlation with $M_{V}$. Our samples, UGC 5981 and UGC 6614, are marked by filled circles. Plotted together are data from various literatures (Kissler-Patig et al. 1999; Goudfrooij et al. 2003; Rhode \& Zepf 2003; Chandar et al. 2004; Barmby et al. 2006; Rhode et al. 2007; Proctor et al. 2008; Villegas et al. 2008; Mora et al. 2009; Harris et al. 2009; Rhode et al. 2010).

galaxies is 0.51. Although it is not plausible to have as a low value as 0.1 for these late-type galaxies, LSBGs have certainly lower values than their HSB counterparts.

Therefore, it is puzzling that disk galaxies with very well-developed bulges, especially, UGC 6614, an Sa type galaxy, have only a couple tens of GCs. If these galaxies have formed just as their higher surface brightness counterparts, their SFHs should be similar to those of HSB galaxies. This is not what we see with our detection of GCSs from our sample. The plausible explanation can be drawn from the scenario described by Kim (2007) which is elaborated by Kim et al. (in 
preparation). LSBGs generally have higher specific star formation rates while having great amount of gas reservoirs intact. Either they simply form very recently due to late gas collapse from primordial density fluctuation, or they accrete neighboring gas continually. Based on rather substantial stellar masses of these galaxies, the latter case is more plausible. Then, if these LSB galaxies experience sporadic star formation activities strong enough to form GCSs along their life times albeit pretty weaker than their HSB counterparts, they should have fewer GCSs which have a wide range of age and, possibly metallicity. Although it requires to perform spectroscopic follow-up study in order to confirm our GC candidates, the nature of our GC candidates fits with the scenario.

\section{SUMMARY}

We have studied the GCSs of two face-on low surface brightness spiral galaxies, UGC 5981 and UGC 6614 . We have located 12 and 18 GC candidates for UGC 5981 and UGC 6614, respectively. Upon extrapolating the estimation, they have a specific frequency within error from the typical values for their corresponding Hubble types, although these values are still much smaller. The estimates values are $S_{N}=0.11$ for UGC 5981 and $S_{N}=0.07$ for UGC 6614. GCSs of LSB galaxies have strong peaks redder than the usual color distribution of GC systems in HSB systems. At this point, it is not obvious that all the redder clusters are the real products of different formation and evolution scenario for low surface brightness galaxies. However, it is plausible that their relatively low specific frequencies reflect their SFHs which has an ephemeral nature.

\section{ACKNOWLEDGMENTS}

The author sincerely appreciates the anonymous referee for helpful comments and suggestions to improve the quality of this draft. He also thanks Stacy McGaugh for endless support and for encouraging me to publish this work. This work was supported by the National Research Foundation of Korea(NRF) grant funded by the Korean government(MEST), No. 20090063616 .

\section{REFERENCES}

Ashman, K. M., \& Zepf, S. E. 1992, The formation of Globular Clusters in Merging and Interacting Galaxies, ApJ, 384, 50

Ashman, K. M., \& Zepf, S. E. 1998, Globular Cluster Systems (Cambridge, U. K. : Cambridge University Press)

Barmby, P., Kuntz, K. D., Huchra, J. P., \& Brodie, J. P. 2006, Hubble Space Telescope Observations of Star Clusters in M101, AJ, 132, 883

Bertin, E., \& Arnouts, S. 1996, SExtractor: Software for Source Extraction, A\&AS, 117, 393
Bothun, G. D., Impey, C. D., Malin, D. F., \& Mould, J. R. 1987, Discovery of a Huge Low-SurfaceBrightness Galaxy - A Protodisk Galaxy at Low Redshift?, AJ, 94, 23

Bothun, G. D., Schombert, J. M., Impey, C. D., Sprayberry, D., \& McGaugh, S. S. 1993, The Small Scale Environment of Low Surface Brightness Disk Galaxies, AJ, 106, 530

Canzian, B. 1998, Extensive Spiral Structure and Corotation Resonance, ApJ, 502, 582

Chaboyer, B., Demarque, P., Kernan, P. J., \& Krauss, L. M. 1996, A Lower Limit on the Age of the Universe, Science, 271, 957

Chandar, R., Whitmore, B., \& Lee, M. G. 2004, The Globular Cluster Systems of Five Nearby Spiral Galaxies: New Insights from Hubble Space Telescope Imaging, ApJ, 611, 220

de Blok, W. J. G., \& van der Hulst, J. M. 1998, Star Formation and the Interstellar Medium in Low Surface Brightness Galaxies. I. Oxygen Abundances and Abundance Gradients in Low Surface Brightness Disk Galaxies, A\&A, 335, 421

de Vaucouleurs, G., de Vaucouleurs, A., Corwin, H. G., Buta, R. J., Paturel, G., \& Fouque, P. 1991,Third Reference Catalogue of Bright Galaxies, (New York : Springer-Verlag) (RC3)

Elson, R. A. W., \& Fall, S. M. 1985, A Luminosity Function for Star Clusters in the Large Magellanic Cloud, PASP, 97, 692

Goudfrooij, P., Alonso, M. V., Maraston, C., \& Minniti, D. 2001, The Star Cluster System of the 3-GyrOld Merger Remnant NGC 1316: Clues from Optical and Near-Infrared Photometry, MNRAS, 328, 237

Goudfrooij, P., Strader, J., Brenneman, L., KisslerPatig, M., Minniti, D., \& Edwin Huizinga, J. 2003, Hubble Space Telescope Observations of Globular Cluster Systems along the Hubble Sequence of Spiral Galaxies, MNRAS, 343, 665

Harris, W. E., \& van den Bergh, S. 1981, Globular Clusters in Galaxies beyond the Local Group. I New Cluster Systems in Selected Northern Ellipticals, AJ, 86, 1627

Harris, W. E. 1991, Globular Cluster Systems in Galaxies beyond the Local Group, ARA\&A, 29, 543

Harris, W. E., \& Pudritz, R. E., 1994, Supergiant Molecular clouds and the Formation of Globular Cluster Systems, ApJ, 429, 177

Harris, W. E., Mouhcine, M., Rejkuba, M., \& Ibata, R. 2009, Globular Cluster Candidates in NGC 891, MNRAS, 395, 436

Holtzman, J. A., Burrows, C. J., Casertano, S., et al. 1995, The Photometric Performance and Calibration of WFPC2, PASP, 107, 1065 
Holwerda, B. W., Gonzalez, R. A., Allen, R. J., \& van der Kruit, P. C. 2005, The Opacity of Spiral Galaxy Disks. IV. Radial Extinction Profiles from Counts of Distant Galaxies Seen through Foreground Disks, AJ, 129, 1396

Impey, C., \& Bothun, G. 1989, Malin 1 - A Quiescent Disk Galaxy, ApJ, 341, 89

Impey, C. D., Sprayberry, D., Irwin, M. J., \& Bothun, G. D. 1996, Low Surface Brightness Galaxies in the Local Universe. I. The Catalog, ApJS, 105, 209

Kim, J. H. 2007, The Star Formation History of Low Surface Brightness Galaxies, Ph.D. Thesis,

Kissler-Patig, M., Ashman, K. M., Zepf, S. E., \& Freeman, K. C. 1999, HUBBLE SPACE TELESCOPE Imaging of Globular Clusters in the Edge-on Spiral Galaxies NGC 4565 and NGC 5907, AJ, 118, 197

Knezek, P. M. 1993, The Stellar and Gaseous Content of Massive Low surface Brightness Disk Galaxies, Ph.D. thesis, Univ.of Massachusetts

Koekemoer et al. 2002, "HST Dither Handbook", Version 2.0 (Baltimore, STScI)

Krist, J., \& Hook, R. 1997, The Tiny Tim User Manual, Version 4.4 (Baltimore; STScI)

Larson, R. 1992, Galaxy Formation and Evolution, Star Formation in Stellar Systems, eds. Tenorio-Tagle, G., Prieto, M., \& Sanchez, F. (Cambridge, U. K. : University of Cambridge Press) 125

Materne, J. 1978, The Structure of nearby Clusters of Galaxies - Hierarchical Clustering and an Application to the Leo Region, A\&A, 63, 401

McGaugh, S. S. 1992, The Physical Properties of Low Surface Brightness Galaxies, Ph.D. thesis, Univ.of Michigan

McGaugh, S. S. 1994, Oxygen Abundances in Low Surface Brightness Disk Galaxies, ApJ, 426, 135

McGaugh, S. S., \& Bothun, G. D. 1994, Structural Characteristics and Stellar Composition of Low Surface Brightness Disk Galaxies, AJ, 107, 530

Meurer, G. R., Heckman, T. M., Leitherer, C., Kinney, A., Robert, C., \& Garnett, D. R. 1995, Starbursts and Star Clusters in the Ultraviolet, AJ, 110, 2665

Miller, B. W., Whitmore, B. C., Schweizer, F., \& Fall, S. M. 1997, The Star Cluster System of the Merger Remnant NGC 7252, AJ, 114, 2381

Miller, B. W., Lotz, J. M., Ferguson, H. C., Stiavelli, M., \& Whitmore, B. C. 1998, The Specific Globular Cluster Frequencies of Dwarf Elliptical Galaxiesfrom the Hubble Space Telescope, ApJ, 508, L133

Mo, H. J., McGaugh, S. S., \& Bothun, G. D. 1994, Spatial Distribution of Low-Surface-Brightness Galaxies, MNRAS, 267, 129

Mora, M. D., Larsen, S. S., Kissler-Patig, M., Brodie, J. P., \& Richtler, T. 2009, Imaging of Star Clusters in Unperturbed Spiral Galaxies with the Advanced Camera for Surveys. II. A Comparison of Star Cluster Systems in Five Late Type Spirals, A\&A, 501, 949

Nilson, P. 1973, Uppsala General Catalogue of Galaxies (Uppsala, Sweden : Uppsala Astronomical Observatory)

Peebles, P. J. E., \& Dicke, R. H. 1968, Origin of the Globular Star Clusters, ApJ, 154, 891

Peterson, C. J. 1993, Integrated Photometric Properties of Globular Clusters, Structure and Dynamics of Globular Clusters, 50, 337

Proctor, R. N., Forbes, D. A., Brodie, J. P., \& Strader, J. 2008, Keck Spectroscopy of Globular Clusters in the Spiral Galaxy NGC 2683, MNRAS, 385, 1709

Puzia, T. H., \& Sharina, M. E. 2008, VLT Spectroscopy of Globular Clusters in Low Surface Brightness Dwarf Galaxies, ApJ, 674, 909

Rhode, K. L., \& Zepf, S. E. 2003, The Globular Cluster System of the Spiral Galaxy NGC 7814, AJ, 126, 2307

Rhode, K. L., Zepf, S. E., Kundu, A., \& Larner, A. N. 2007, Global Properties of the Globular Cluster Systems of Four Spiral Galaxies, AJ, 134, 1403

Rhode, K. L., Windschitl, J. L., \& Young, M. D. 2010, WIYN Imaging of the Globular Cluster Systems of the Spiral Galaxies NGC 891 and NGC 4013, AJ, 140,430

Schombert, J., Bothun, G. D. Schneider, S. E., \& McGaugh, S. S. 1992, A Catalog of Low Surface Brightness Galaxies - List II, AJ, 103, 1107

Schweizer, F. 1997, Mergers and Globular-Cluster Formation in Early-Type Galaxies, The Nature of Elliptical Galaxies; 2nd Stromlo Symposium, 116, 447

Searle, L., \& Zinn, R. 1978, Compositions of Halo Clusters and the Formation of the Galactic Halo, ApJ, 225,357

Sharina, M. E., Puzia, T. H., \& Makarov, D. I. 2005, Hubble Space Telescope Imaging of Globular Cluster Candidates in Low Surface Brightness Dwarf Galaxies, A\&A, 442, 85

Villegas, D., Kissler-Patig, M., Jordán, A., Goudfrooij, P., \& Zwaan, M. 2008, Normal Globular Cluster Systems in Massive Low Surface Brightness Galaxies, AJ, 135, 467

Whitmore, B. C., \& Schweizer, F. 1995, Hubble Space Telescope Observations of Young Star Clusters in NGC-4038/4039, 'the Antennae' Galaxies, AJ, 109, 960

Whitmore, B. C., Miller, B. W., Schweizer, F., \& Fall, S. M. 1997, Hubble Space Telescope Observations of Two Dynamically Young Elliptical Galaxies, AJ, 114,1797 